\title{
Aspects of programmable calculator stimulus control and data acquisition in a psychophysiology laboratory
}

\author{
RICHARD M. BLOCH \\ Eastern State Hospital and the College of William and Mary, Williamsburg, Virginia 23185
}

\begin{abstract}
Advantages and disadvantages of programmable calculator control of research laboratories are discussed. Comparisons are drawn with microcomputers such as the Imsai 8080 . While microcomputers have greater flexibility in sampling multiple measures at multiple rates and combining timing tasks with data acquisition, calculators are easier to program for applications combining data acquisition with mathematical processing. Calculators also offer advantages to laboratories that do not have access to programming or maintenance services.
\end{abstract}

Microprocessor-based computers, including some programmable calculators, have tremendous potential impact on the automation of laboratory processes in psychology. There is a great deal of overlap in capabilities among the microprocessor-based computers, although there are some research environments for which programmable calculators, such as the HewlettPackard 9825, Monroe 1860, Tektronix 31, and Wang PCS Il, may be better suited than more flexible microcomputers, such as the Imsai 8080 , SWTPC 6800 , KIM I, and so on. This paper briefly discusses general and somewhat arbitrary differences between these programmable calculators and popular microcomputers. Aspects of the application of one of the calculators, a Monroe 1860, to stimulus control and data acquisition in a psychophysiology laboratory are also discussed. The result will be to establish programmable calculators, and thus microprocessor-based computers in general, as alternatives to both hardwired logic modules and minicomputers, and to describe situations for which either programmable calculators or microcomputers might be best suited for laboratory control.

Programmable calculators are designed with mathematical and statistical operations in mind and are packaged with all the peripherals, read-only memory (ROM) mathematical subroutines, and ROM-stored higher level language necessary to enable immediate entry, processing, and mass storage of programs and data. Each machine has an integral keyboard, often alphanumeric, with user-definable keys, and instructions and system-control keys that call ROM subroutines with a single keystroke. Each machine contains at least one visual display method; either a printer, a cathoderay tube (CRT), and/or a multiple-character light display. Each machine also contains a mass storage device such as a magnetic card reader, a tape cartridge, or a floppy disk.

Microcomputers are designed to be more modular and flexible. The base machines often contain no interfaced peripherals, or have either a front panel with toggle switches and lights or a hexidecimal key pad and six-character light display. Only the machine language operating system is stored in ROM. However, a wide variety of peripherals are available, including alphanumeric keyboards, printers, tape cartridges, and floppy disks. The user chooses the peripherals best suited to a particular application. Higher level languages or mathematical subroutines can be read or entered into random access memory (RAM) when needed. If these routines are not needed, all memory is available for other applications.

These differences in design philosophy have mixed effects on costs. The base prices for microcomputers are considerably less expensive than for programmable calculators. Of course, adding comparable peripherals and memory to the microcomputers diminishes cost differences. Further, memory expansion is much less expensive and more peripherals are available from a wider variety of sources for microcomputers. However, hardware support for the microcomputer and peripherals is more costly in time, if not money. Almost every major city has sales and service centers for programmable calculators. Most problems with the processor or integral peripherals are repaired within 1 week, and "loaners" are often available while the machine is being repaired. Microcomputer and most minicomputer companies do not have such extensive service facilities and are not responsible for peripherals purchased from other companies. Thus, repairs are almost always slower and often more expensive.

Programmable calculators are often easier to lease toward purchase than other computers. There may be little or no additional cost for calculators purchased through several payments across fiscal years. Other computers often cannot be leased without doubling the price. 


\section{PROGRAMMABLE CALCULATORS IN A PSYCHOPHYSIOLOGY LABORATORY}

In part, for these reasons, a Monroe 1860 programmable calculator was employed to control on- and off-line data acquisition and stimulus presentation in a psychophysiology laboratory. The 1860 is not a "state-of-the-art" programmable calculator and of the calculators listed it has the slowest execution time for machine language instructions, nominally 80 microsec. This is also far slower than the instruction times for microprocessors such as the 8080,6800 , or 6502 .

Because of the slow instruction time, all input and output (I/O) functions must use machine language rather than the higher level language stored in ROM. Maximum I/O transfer rates are then 3,000 bytes $/ \mathrm{sec}$ or less, unless direct memory access (DMA) is employed. However, this speed is adequate for sampling typical psychophysiological systems such as the electroencephalogram (EEG), the electromyogram (EMG), skin resistance (GSR), and the electrooculogram (EOG). To digitize these measures, an eight-channel multiplexer and 8-bit analog-to-digital (A/D) converter are interfaced to the calculator. The multiplexer allows simultaneous sampling of multiple-response systems, or of multiple leads from the same system. For instance, sampling each channel every $4 \mathrm{msec}$, eight leads of EEG can be simultaneously digitized for mapping cortical evoked response or contingent negative variation (CNV) distributions over the scalp. Slower response systems, such as GSR and EOG, can also be sampled and scored without difficulty.

In terms of 8-bit A/D conversion, the Monroe 1860 has sufficient speed to sample measures commonly used in psychophysiology laboratories. However, for investigations requiring more than 8-bit resolution, maximum sampling rate falls to approximately $1,750 / \mathrm{sec}$. If an application requires digitizing multiple measures at different rates, for example, rates of 200 and 500 samples/sec, the processor must be able to sample at the combined rate, that is, 1,000 samples $/ \mathrm{sec}$. A maximum sampling rate of $1,750 / \mathrm{sec}$ markedly reduces the range of measures and rates that can be sampled simultaneously. The higher speeds of the microcomputers, and to a lesser extent the other calculators, allow greater flexibility in sampling at multiple rates using either 8 - or 10 -bit A/D converters.

Interval timing measures such as reaction time and heart period, which can be converted to heart rate, have also been obtained with the Monroe 1860. The calculator does not have sufficient speed, in checking input level lines for heart beat or button press occurrence, to measure these intervals to the nearest millisecond. However, by sampling once each millisecond starting near $.5 \mathrm{msec}$, rounding to the nearest millisecond is closely approximated.

The fixed instruction time in the Monroe 1860 makes timing loops easier and thus facilitates programming these timing operations. However, time measurements to the nearest millisecond require dedicated processing and do not allow other simultaneous functions, such as data acquisition or manipulation.

Many control operations also require timing, such as calculator initiation of stimulus onset and offset with program control of stimulus duration, interstimulus interval, and intertrial interval. A peripheral clock with program-selectable frequencies increases the ability of the calculator to control these parameters and simultaneously acquire and process data. Addition of such a clock is not needed with microcomputers that contain one or more programmable interval timers.

Data processing is facilitated by the 1860 's ROM arithmetic routines and higher level language, which can be mixed with machine language instructions. Further, these routines can reduce program size. For example, a routine converting pre- and poststimulus resistance levels to change in log conductance and printing the answer rounded to any decimal place up to eight requires only 10 instruction steps or bytes of RAM. The ROM routines can operate on hexidecimal as well as binary-coded decimal (BCD) information, although data must be converted to $\mathrm{BCD}$ before printing. Thus, on-line signal averaging operations sum 8-bit binary samples, average them, and then convert the averages to $\mathrm{BCD}$ for printing or plotting via a peripheral digital-to-analog converter.

The internal organization of the calculator also facilitates mathematical operations. Unlike microcomputers, which generally contain 8- or 16-bit accumulators, the calculator has a 64-bit accumulator with additional overflow and underflow bits. Unlike microcomputers, calculator RAM can be treated in 8-bit program steps or 8-byte (64-bit) registers. Thus, single-byte machine language instructions in the 1860 can store, recall, or manipulate 8 bytes of program or data information simultaneously. Compared to microcomputers, which must read BASIC into RAM for extended arithmetic operations, programs using the single-byte arithmetic instructions in the Monroe require fewer bytes of RAM and may even have comparable execution times.

To summarize, the Monroe 1860 is one example of programmable calculators that have sufficient speed and flexibility to be employed as laboratory computers. They can perform all but the most complex control and data acquisition tasks, and thus can be considered, along with the microcomputers, as alternatives to hardwired logic modules and minicomputers for most laboratory situations. The microcomputers' greater flexibility and ability to be individually configured to fit research requirements make them the better alternative in a wider variety of situations. Microcomputers' faster instruction times and internal interval timers make them most appropriate for complex timing 
applications and for fast sampling of multiple measures at multiple rates. The greater expandability of microcomputer memory and wider array of peripherals available make these machines more adaptable to originally unanticipated applications. However, laboratories with little electronics maintenance support and/or with insufficient funds to afford extended interruptions waiting for repairs may find a programmable calculator to be the computer of choice. Calculators may also be reasonable for situations where there is little programming help and where mathematical operations are frequent. The integration of peripherals and processor into a single compact unit makes calculators more suitable where portability is important. Finally, when there are insufficient funds available in any one fiscal year to purchase a complete microcomputer configuration, leasing a programmable calculator may allow laboratory automation with minimal delay. 